\title{
Detección de drogas en saliva: aspectos metodológicos y legales
}

\section{Detection of drugs in saliva: methodological and legal aspects}

\section{Resumen}

El análisis de drogas en saliva es una alternativa no invasiva para evidenciar su presencia en el organismo. La finalidad es amplia, pero se ha utilizado en medicina legal y forense, en especial para drogas ilícitas. La recolección de saliva no implica riesgo, ni personal especializado. El objetivo fue valorar diversos aspectos metodológicos y de validez del test de drogas en saliva para el diagnóstico de su consumo. Asimismo, se analizaron algunos aspectos relativos a la legislación de drogas. Las sustancias psicoactivas a evaluar fueron marihuana, cocaína, anfetaminas y opiáceos. Se realizó una búsqueda en las bases de Pubmed/MEDLINE, Embase, LILACS, Scopus, SciELO y de literatura gris. La identificación de drogas en fluidos biológicos incluye técnicas inmunoquímicas, cromatográficas y procedimientos espectrométricos. Resultados positivos obtenidos mediante una técnica deberían confirmarse con un segundo análisis basado en distintos principios químicos o físicos. Se han detectado en saliva marihuana, cocaína, opiáceos y anfetaminas, entre otras, mediante métodos analíticos, como inmunoensayo y cromatografía de gases / espectrometría de masas en tándem. En el fluido oral, se pueden detectar drogas de abuso durante 5-48 horas posteriores al consumo, a un nivel bajo de nanogramos por mililitro. Se concluyó que la detección de drogas en saliva es una opción indicada ante sospecha de su consumo, por signos o síntomas que sugieren abuso de drogas o para la vigilancia del usuario en un centro de tratamiento por dependencia. En el aspecto legal el uso de test de drogas en saliva es una posibilidad para determinadas circunstancias.

Palabras clave: Drogas ilícitas; Saliva; Diagnóstico; Legislación de drogas (fuente: DeCS BIREME).

\begin{abstract}
The analysis of drugs in saliva is a non-invasive alternative to demonstrate their presence in the body. The purpose is broad, but it has been used in forensic and legal medicine, especially for illicit drugs. The collection of saliva is not risky and does not require specialized personnel. The objective was to assess various methodological and validity aspects of the saliva drug test for the diagnosis of drug use. Likewise, some aspects related to drug legislation were analyzed. The psychoactive substances to be evaluated were marijuana, cocaine, amphetamines and opiates. A search was carried out
\end{abstract}

\section{Artículo de Revisión}

Enrique Rotemberg 1,a, Alicia Picapedra 1,b, Marcelo Kreiner ${ }^{1, c}$

${ }^{1}$ Universidad de la República, Facultad de Odontología, Montevideo, Uruguay.

a Magister en Ciencias Odontológicas.

${ }^{\mathrm{b}}$ Doctorado en Salud Colectiva.

' Doctorado en Ciencias Médicas.

\section{Correspondencia:}

Enrique Rotemberg: erotemb@yahoo.com.ar General las Heras 1925, Facultad de Odontología, Udelar, Uruguay. Código postal: 11600 Montevideo. ORCID: 0000-0002-0686-8607

\section{Coautores:}

Alicia Picapedra: almapipa@gmail.com ORCID: 0000-0003-0166-1145

Marcelo Kreiner: mkreiner@netgate.com.uy ORCID: 0000-0001-6463-1280

\section{Editora:}

María Eugenia Guerrero

Universidad Nacional Mayor de San Marcos, Perú.

Conflicto de intereses: los autores declaran no tener conflictos de interés.

Fuente de financiamiento: autofinanciado

Recibido: 05/08/21

Aceptado: 24/09/21

Publicado: 21/01/22 
in the Pubmed / MEDLINE, Embase, LILACS, Scopus, SciELO and gray literature databases. Identification of drugs in biological fluids was found to include immunochemical, chromatographic and spectrometric procedures. Positive results obtained by one technique must be confirmed with a second analysis based on different chemical or physical principles. Marijuana, cocaine, opiates and amphetamines, among other drugs, have been detected in saliva by various analytical methods, like immunoassay and gas chromatography / tandem mass spectrometry. In oral fluid, drugs of abuse can be detected for 5-48 hours post-consumption, at a low level of nanograms per milliliter. It was concluded that the detection of drugs in saliva is an option that is indicated in the event of suspected drug use, for signs or symptoms that suggest drug abuse, or for monitoring the user in a dependency treatment center. In the legal aspect, the use of drug tests in saliva is a possibility for certain circumstances.

Keywords: Illicit drugs; Saliva; Diagnosis; Legislation drug (source: MeSH NLM).

\section{Introducción}

El consumo voluntario o accidental de drogas se puede detectar mediante marcadores de esas drogas en muestras biológicas como orina, saliva y sangre ${ }^{1}$. El análisis de muestras de fluido oral (saliva mixta o total) se usa cada vez más para detectar el uso reciente de drogas ${ }^{2}$. La saliva es una matriz alternativa viable para identificar la presencia de sustancias psicoactivas (SPA) como tetrahidrocannabinol (THC), cocaína (COC), anfetaminas (AMP) y opioides (OPI) ${ }^{3}$. La saliva mixta es una solución acuosa de predominio ácido compuesta por secreciones de las glándulas salivales principales o mayores (parótida, submaxilar y sublingual) y de glándulas accesorias o menores (labiales, yugales, linguales, palatinas y faríngeas), líquido gingival crevicular, microorganismos, secreciones nasales y bronquiales, células epiteliales de revestimiento, sangre en individuos con gingivitis y sustancias exógenas ${ }^{4}$.

Los test de drogas podrían ser indicados por motivos de seguridad vial, laborales, dopaje en el deporte, post-morten o porte de armas ${ }^{5}$. La incorporación de drogas y/o sus metabolitos al fluido oral puede ser durante la masticación si la droga es ingerida, al fumar si es inhalada o ingresando a la saliva total desde el torrente sanguíneo ${ }^{6}$. La sangre y la orina son los fluidos de diagnóstico más utilizados en entornos de laboratorio, a pesar de que la saliva tiene algunas ventajas en comparación con dichas matrices biológicas. Por ejemplo, la recolección de saliva es fácil de realizar, no invasiva y requiere instrucciones de colección relativamente simples ${ }^{7}$.

La extracción de la muestra de fluidos orales se puede realizar en casi cualquier lugar en condiciones directamente observadas, con escasa probabilidad de adulteración y/o sustitución ${ }^{8}$. Se han desarrollado técnicas cromatográficas para detectar y cuantificar múltiples clases de fármacos en fluidos orales ${ }^{9}$. Los fármacos están presentes en niveles más bajos en saliva en relación a la orina ${ }^{10}$.

El objetivo general fue valorar diversos aspectos metodológicos, legales y de validez del test de drogas en saliva para la identificación de las sustancias psicoactivas marihuana, cocaína, anfetaminas y opiáceos. Además se analizaron las técnicas viables de test de drogas en saliva con la finalidad de detectar la exposición a determinadas sustancias de abuso o de sus metabolitos en dicho fluido biológico y examinar normas de la legislación nacional vigente para el control del consumo de sustancias psicoactivas.

\section{Métodos}

Se realizó la presente revisión narrativa mediante la búsqueda de información en las siguientes bases de datos: Pubmed/MEDLINE, Embase, LILACS, Scopus, SciELO y bases de literatura gris, empleando como palabras clave los Descriptores en Ciencias de la Salud (DeCS): drogas ilícitas, saliva, diagnóstico y legislación de drogas, y sus correspondientes Medical Subject Headings $(\mathrm{MeSH})$ illicit drugs; saliva; diagnosis; legislation, drug, y como operadores booleanos and, not y or. Se incluyeron artículos originales y de revisión que trataron sobre la efectividad de los test de drogas que utilizan fluidos orales, la comparación con el uso de otras matrices biológicas y aspectos legales relacionados a su indicación. Se excluyeron artículos referidos al consumo de alcohol, tabaco, cafeína u otras drogas o fármacos no comprendidos en el presente artículo. Los idiomas seleccionados fueron español e inglés.

\section{Reseña de las drogas analizadas}

El cannabis, conocido también como marihuana, se fuma como droga de uso recreativo, aunque puede ańadirse a los alimentos ${ }^{11}$. La principal sustancia psicoactiva de la marihuana es el delta-9-tetrahidrocannabinol $\left(\Delta 9\right.$-THC) ${ }^{12}$. El $\Delta 9$-THC ejerce sus efectos sobre 2 tipos de receptores cannabinoides de membrana específicos acoplados a proteína G (RCB1 y RCB2) y un ligando endógeno, la anandamida ${ }^{13}$. Los RCB1 se localizan en células del sistema nervioso central (SNC) y los RCB2 se encuentran principalmente en células del sistema inmune ${ }^{14}$.

Asimismo, se ha demostrado la existencia de un sistema cannabinoide endógeno definido como un sistema de señalización lipídica presente en todos los vertebrados, que cumple importantes funciones de regulación. El sistema endocannabinoide (SEC) ha sido involucrado en procesos fisiológicos y fisiopatológicos como: desarrollo neural, función inmune, inflamación, apetito, metabolismo 
energético, plasticidad sináptica, reproducción, memoria, ciclo sueño-vigilia y regulación del estrés entre otros. Por lo tanto, los cannabinoides presentes en la planta de cannabis modulan dichos procesos actuando sobre receptores del SEC ${ }^{15}$. El consumo de marihuana afecta a casi todos los sistemas del cuerpo, particularmente los sistemas cardiovascular, respiratorio e inmunológico. También tiene efectos agudos y crónicos en el estado de salud mental de algunos usuarios ${ }^{11}$.

La cocaína (COC) pura era extraída originalmente de la hoja del arbusto de la coca del género Erythroxylum. El consumo tradicional de $\mathrm{COC}$ en pueblos indígenas de América es de origen ancestral, con el fin de atenuar el hambre y la fatiga. Más recientemente su uso como anestésico local se restringió por su toxicidad y riesgo de adicción ${ }^{16}$. La COC produce efectos desde la etapa prenatal, pasando por la niñez, la adolescencia y la vida adulta. El consumo de COC único o combinado con alcohol formando cocaetileno, puede producir la muerte ${ }^{13}$. Este metabolito prolonga la sensación de euforia, produce mayor depresión miocárdica y aumenta la vida media en 2,5 veces respecto a la COC sola, lo que puede dar lugar a manifestaciones clínicas tardías ${ }^{16}$.

Las anfetaminas (AMP) son un grupo de drogas estimulantes del SNC. Bajo ese término se engloban diversas sustancias como la anfetamina propiamente dicha, la dextranfetamina, la metanfetamina y los estimulantes tipo anfetamina: metilfenidato, MDA (3,4-metilenodioxianfetamina), MDMA (3-4, metilenodioximetanfetamina, "éxtasis"), entre otros ${ }^{17}$. Los consumidores crónicos de metanfetaminas pueden presentar síntomas que incluyen ansiedad intensa, confusión, insomnio, cambios del estado de ánimo y comportamiento violento. También pueden manifestar una serie de trastornos psicóticos como paranoia, alucinaciones visuales y/o auditivas y delirios ${ }^{18}$. La AMP se considera una droga xerogénica y los consumidores de esta droga a menudo se quejan de sequedad bucal ${ }^{19}$.

Los opioides (OPI) son sustancias con afinidad por los receptores opioides del SNC. Incluye sustancias endógenas y exógenas, naturales o sintéticas. El opio se extrae de un tipo de amapola llamada Papaver somniferum. Su uso altera la percepción del dolor produciendo analgesia, aunque puede causar tolerancia y farmacodependencia ${ }^{16}$. Los signos clínicos característicos conforman una tríada que se reconoce por: depresión respiratoria, disminución de la conciencia y miosis (pupila puntiforme) ${ }^{13}$. Los OPI u opiáceos comprenden la heroína, el fentanilo, la oxicodona, la hidrocodona, la codeína y la morfina entre otros. El consumo regular, aún con indicaciones del médico, puede llevar a la dependencia. La sobredosis de OPI puede causar la muerte, aunque se puede revertir con la droga naloxona si se administra en forma inmediata ${ }^{20}$. Los OPI, al igual que las AMP y el alcohol son xerostómicos, reducen la producción de saliva ${ }^{21}$.

\section{Criterios a valorar del análisis de laboratorio}

Cuando se realiza un análisis de laboratorio sencillo (dicotómico), se deben valorar dos opciones: negativo (no detectable, ausente) / positivo (detectable, presente), términos referidos para indicar la presencia o ausencia de la condición bajo estudio. La sensibilidad es la proporción de verdaderos positivos que son detectados por la prueba. Es recomendable que los análisis presuntivos sean de alta sensibilidad, ya que aunque pueden incluir un porcentaje de falsos positivos, se requiere captar el mayor número de individuos positivos, enfermos y/o con la condición de interés.

Otro parámetro que se puede evaluar es la especificidad, se refiere a la proporción de verdaderos negativos captados en los test. Los valores de sensibilidad y especificidad para una variable estadística dicotómica binaria como lo es la detección de drogas en fluidos biológicos, se complementan con los resultados de falsos negativos y falsos positivos respectivamente (Tabla $1{ }^{2}{ }^{22}$. Los resultados negativos de los test no dan seguridad de falta de consumo de SPA, en cambio los resultados positivos reflejan el consumo de una o más SPA ${ }^{23}$. El límite de detección es la mínima cantidad o concentración de SPA que puede ser detectada por un método analítico ${ }^{22}$.

\section{Métodos de identificación de drogas}

La metodología utilizada para la detección de drogas en fluidos biológicos a lo largo de la historia ha sido a través de técnicas cromatográficas, fluorometría, microcristalografía, métodos colorimétricos, procedimientos espectrométricos, métodos de fluorescencia, radioinmunoanálisis y enzimoinmunoanálisis ${ }^{24,25}$. Todos los resultados positivos obtenidos mediante una detección por inmunoanálisis deberían confirmarse con un segundo análisis basado en distintos principios químicos o físicos como las técnicas cromatográficas. Las pruebas de confirmación deberían ser de similar sensibilidad aunque de mayor especificidad que las pruebas de detección ${ }^{26}$.

La marihuana, la cocaína, los opiáceos y las anfetaminas entre otras drogas se han detectado en la saliva mediante varios métodos analíticos, incluidos el inmunoensayo "immunoassay", la cromatografía de gases / espectrometría de masas "gas chromatography/mass spectrometry" (GC-MS) y la cromatografía en capa fina "thin layer

Tabla 1. Criterios a valorar de análisis de laboratorio

\begin{tabular}{lll}
\hline Análisis dicotómico de drogas en fluidos & Negativo: no detectable & Positivo: detectable \\
\hline Sensibilidad & Baja: alto porcentaje de falsos negativos & Alta: elevado porcentaje de verdaderos positivos \\
Especificidad & Baja: alto porcentaje de falsos positivos & Alta: elevado porcentaje de verdaderos negativos \\
\hline
\end{tabular}


chromatography" (TLC) ${ }^{27}$. Los inmunoensayos cuantifican moléculas de interés biológico según la especificidad y selectividad de los reactivos de anticuerpos generados ${ }^{28}$. La GC-MS es una técnica que combina el poder de discriminación y la sensibilidad de la cromatografía de gases (GC) con la especificidad para la muestra analizada que aporta la técnica espectroscópica, sin necesidad de aislar previamente las sustancias a detectar. La TLC es una técnica utilizada habitualmente para la separación e identificación de drogas ilícitas, al ser una técnica económica, rápida, sensible y que requiere cantidades inferiores al miligramo de la sustancia objeto de análisis ${ }^{29}$.

El método más utilizado para el estudio toxicológico de AMP es la cromatografía líquida asociada a espectrometría de masas (LC-MS) (liquid chromatographymass spectrometry), tanto en muestras en sangre como en otro tipo de sustratos, incluyendo la saliva. Es una técnica precisa, estable y que permite realizar detección de múltiples drogas al mismo tiempo ${ }^{17}$. Entre los ensayos in vitro para la detección de anticuerpos, el ELISA (Enzyme-Linked-Immunosorbent Assay o ensayo por inmunoabsorción ligado a enzimas) se caracteriza por su precisión y exactitud, sin los riesgos que implica el empleo de material radioactivo como con el radioinmunoanálisis ${ }^{30}$. La especificidad y sensibilidad de pruebas inmunoenzimáticas en saliva, como ELISA por ejemplo, puede afectarse por varios factores, entre los que se incluyen la ingesta previa de alimentos o bebidas, el lavado de la boca o el uso de pasta de dientes. Esta situación no se da en el caso de LC-MS ${ }^{17}$.

Los tiempos de detección de drogas varían en función de la vía de administración, la dosis y tipo de droga, el consumo esporádico o habitual, la matriz biológica elegida, la sensibilidad del test utilizado y las variaciones individuales según el aclaramiento metabólico y renal ${ }^{31}$.

Según la matriz biológica las SPA a un nivel bajo de nanogramos por mililitro se pueden detectar en sangre 1 o 2 días, en orina 1,5 a 4 días y en fluido oral de 5 a 48 horas posteriores al consumo ${ }^{32}$. La prueba de saliva puede detectar COC o sus metabolitos solo uno o dos días después del consumo. Los dos metabolitos más importantes de la COC son la benzoilecgonina y la ecgonina metil éster. La prueba usualmente detecta el primero de estos dos metabolitos ${ }^{31}$.

El THC inhalado puede ser detectado en la sangre con más rapidez que el ingerido, siendo en este último caso degradado en alto porcentaje por la secreción ácida del estómago ${ }^{33}$. En condiciones extremas (interior del automóvil), se puede observar la contaminación pasiva de THC en la saliva (de un fumador a un no fumador) durante aproximadamente 30 minutos ${ }^{12}$.

\section{Aspectos legales en el consumo de SPA}

En Uruguay, el marco normativo que refiere a la detección de drogas en fluidos corporales, incluye dos leyes de tránsito y seguridad vial y un decreto reglamentario laboral. La Ley No 18.191 del 14 de noviembre de 2007, en sus artículos 45 y 46, establece la prohibición para conducir en el territorio nacional frente a concentraciones de alcohol u otras drogas psicotrópicas, habilitando el control de cualquier conductor, mediante procedimientos de espirometría estipulados por las autoridades competentes y ratificados por exámenes clínicos o paraclínicos, de sangre u orina ${ }^{34}$. En 2015, la Ley No 19.360 modifica el artículo 45, inhabilitándose a los conductores con presencia de tetrahidrocannabinol (THC) o cocaína en el organismo y sustituye el artículo 51, incorporando a la saliva como un elemento a ser examinado, posibilitando al conductor solicitar a las autoridades del Ministerio de Salud Pública la realización de otros exámenes, para ratificar o rectificar los resultados precedentes ${ }^{35}$.

Con la aprobación del Decreto 128/016 entró en vigencia una norma que reglamenta, en su artículo 2 el consumo de alcohol y drogas en el ambiente laboral, aplicándose a toda relación de trabajo, ya sea en el sector público o privado, y prohibiendo el consumo y/o tenencia de cualquier tipo de droga en los lugares de trabajo o en ocasión del mismo, debiéndose detectarlas conforme al protocolo acordado entre la empresa y el sindicato, en los ámbitos bipartitos de seguridad y salud. Será potestad del empleador pedir las pruebas de un eventual consumo, mediante dispositivos analíticos no invasivos. Todo trabajador con resultado positivo en las pruebas de despistaje, tendrá derecho a solicitar una prueba confirmatoria en sangre para alcohol; y en saliva para cannabis y cocaína ${ }^{36}$.

\section{Revisión y Discusión}

Ventajas y desventajas del test de drogas en saliva. La saliva refleja el estado de salud general del cuerpo humano y es fácil de recolectar, por lo que puede ser una herramienta de diagnóstico no invasiva ${ }^{37}$. En las muestras de saliva pueden realizarse estudios diversos sobre: neoplasias, drogas recreativas y de abuso (Tabla 2), medicamentos antiepilépticos, antineoplásicos, metales pesados, enfermedades congénitas y metabólicas, ácidos nucleicos entre otros, de gran aplicación en medicina legal, ocupacional y forense. A diferencia de las muestras de sangre las de saliva no requieren personal experimentado para su obtención, el procedimiento no es invasivo ni doloroso, la posibilidad de exposición accidental a patógenos virales y microbianos es prácticamente nula, a diferencia de las muestras de sangre por uso de agujas contaminadas ${ }^{38}$.

Los test de drogas en orina fueron positivos en mayor porcentaje para el consumo simultáneo de drogas en muestras pareadas con los test en saliva. La confirmación de la ingesta simultánea de fármacos es posible mediante el uso fluido oral, pero de menor eficacia respecto a la detección en orina ${ }^{39}$. La orina es reconocida como la matriz principal para la detección de drogas con métodos y protocolos de prueba bien establecidos. Su principal limitación está relacionada con los inconvenientes de la recolección de muestras y la posibilidad de adulteración, dilución, adición de drogas o intercambio de muestras ${ }^{40}$. Las drogas se retienen por un período más 
Tabla 2. Drogas recreativas o de abuso

\begin{tabular}{ll}
\hline Drogas & Origen \\
\hline Cotinina & Metabolito derivado de la nicotina \\
Tiocianato & Producto del cianuro; presente en el humo del tabaco, en la industria, en desechos ambientales y ciertos alimentos \\
Tetrahidrocannabinol & Cannabinoide, componente psicoactivo de la marihuana \\
Cocaína & Elaborada con hojas de la planta de coca \\
Anfetaminas & Agente adrenérgico sintético \\
Barbitúricos & Fármacos que causan relajación y somnolencia \\
Benzodiazepinas & Medicamentos que potencian el efecto inhibitorio del neurotransmisor GABA \\
Opioides & Sustancias que se extraen de la cápsula de la planta del opio \\
Feniciclidina & Droga disociativa usada inicialmente como agente anestésico \\
\hline
\end{tabular}

corto en la saliva respecto a la orina y a menores concentraciones ${ }^{25}$. El análisis de orina ofrece una ventana intermedia de detección (1 a 3 días) mientras que el de cabello ofrece la mayor ventana de detección (7 - 100 o más días) y el de saliva puede ser útil para determinar el consumo de drogas muy reciente (1 a 36 horas) ${ }^{41}$.

Los avances en las técnicas analíticas, en particular la cromatografía vinculada a la espectrometría de masas, están ayudando a promover el análisis de fluidos orales ${ }^{40,42}$. La LC-MS permite el análisis simultáneo de múltiples compuestos no volátiles, lábiles, polares y / o de alto peso molecular en un volumen de fluido oral limitado ${ }^{42}$. Un trabajo realizado con muestras de sangre y de saliva al inicio del estudio y 8 horas después de la ingestión de brownies de cannabis que contenían diferentes dosis de THC (entre 0 y $50 \mathrm{mg}$ ), que utilizó el test ELISA y la LC-MS para medir el THC y sus principales metabolitos, mostró en sangre que las concentraciones de $\Delta$ 9-tetrahidrocannabinol (THC) y 11-hidroxi-THC (11-OH-THC) alcanzaron su punto máximo entre 1,5 y $2 \mathrm{~h}$ después del consumo de brownies, disminuyeron posteriormente de forma gradual y, por lo general, volvieron a los valores iniciales en 8 horas. La detección inicial de THC en el fluido oral fue inmediata y en mayores concentraciones que en la sangre ${ }^{43}$. La LC-MS es un método altamente específico, que combina separación cromatográfica y espectrometría de masas, se puede usar para la confirmación de resultados de inmunoensayos positivos ${ }^{44}$. Las mujeres mostraron concentraciones máximas más altas de THC y todos los metabolitos en sangre total en comparación con los hombres, en parte debido a su menor peso e índice de masa corporal. Después del consumo oral de cannabis, se detectó THC en sangre en forma tardía y en menor concentración en comparación con lo observado con el cannabis inhalado ${ }^{43}$.

Ante la preguntas si es similar la detección de drogas en orina frente a la detección de fluidos orales Martini et al. ${ }^{3}$ realizaron una revisión sistemática sobre drogas como THC, COC, AMP y OPI ilícitos, con trabajos seleccionados entre los años 2003-2009, alcanzando estas conclusiones: a) el fluido oral tiene un gran potencial para la detección inmediata del consumo reciente de marihuana en comparación con la orina; b) en relación con la COC y la metanfetamina, la mayor ventana de detección de drogas se obtiene mediante análisis de orina; c) la saliva no puede reemplazar a la orina para la mayoría de los objetivos de las pruebas de detección de drogas. Las pruebas de drogas en orina realizadas tienen más probabilidad de detectar el uso de drogas ilícitas u otras sustancias que las pruebas de fluidos orales, según el resultado de un ensayo clínico pareado usando test de orina y test de saliva ${ }^{45}$.

El pH de la saliva es ligeramente ácido en relación con la sangre; por lo tanto, los fármacos que son más básicos tienden a estar presentes en mayor concentración en el fluido oral que en la sangre: cocaína, anfetaminas, oxicodona, tramadol, buprenorfina, metadona y fentanilo. Por el contrario, los fármacos ácidos y los fármacos que se unen fuertemente a proteínas como benzodiazepinas, barbitúricos y carisoprodol, tienen concentraciones más bajas en el fluido oral que en la sangre ${ }^{46}$. La determinación de clonazepam mediante cromatografía líquida y extracción en fase sólida parece ser adecuado para el análisis de esta benzodiazepina en el fluido oral, con buenos resultados en cantidades mínimas de solvente ${ }^{47}$.

Debido al bajo volumen de muestra disponible para análisis y las concentraciones de drogas presentes, en relación a la sangre y orina, se necesitan métodos de extracción eficientes y procedimientos de confirmación sensibles para el análisis de rutina de fármacos en el fluido oral ${ }^{46}$. En el presente cuadro se mencionan ventajas y desventajas de los test de drogas en fluido oral en relación al uso de otras matrices biológicas ${ }^{48}$ (Tabla 3).

Legalidad en el uso de test de drogas. La detección de drogas de abuso es de interés en medicina legal y forense para sujetos vivos o cadáveres, en el ámbito laboral, en pacientes en tratamiento de desintoxicación de SPA y en diversos procedimientos legales como la detección de conductores de vehículos bajo los efectos del alcohol o las drogas. En este caso la saliva es una matriz de elección por sus ventajas en la recolección y análisis ${ }^{49}$, considerando que el consumo de SPA puede alterar el volumen y la composición del fluido oral ${ }^{50}$.

El consumo de alcohol, cocaína u otras SPA como cannabis, heroína, psicofármacos provoca perjuicios en el 
Tabla 3. Estudio comparativo del test de drogas en fluido oral

\begin{tabular}{ll}
\hline Ventajas & Desventajas \\
\hline $\begin{array}{l}\text { Prueba menos invasiva y más fácil de obtener que otros medios } \\
\text { biológicos }\end{array}$ & Necesidad de estandarizar el procedimiento de obtención y almacenamiento. \\
$\begin{array}{l}\text { Difícil de adulterar o sustituir al ser obtenida con observación } \\
\text { directa }\end{array}$ & Menor retención de drogas en el tiempo por aclaramiento bucal \\
$\begin{array}{l}\text { Se correlaciona con niveles plasmáticos de la droga } \\
\begin{array}{l}\text { El THC alcanza concentraciones iniciales más altas que en } \\
\text { otros medios }\end{array}\end{array}$ & La elevación del pH salival tiende a reducir la presencia de drogas \\
\hline
\end{tabular}

medio laboral como riesgos de accidentes laborales y no laborales, afectando según la Organización Internacional del Trabajo (OIT) a un porcentaje considerable de trabajadores ${ }^{51}$.

Existe consenso en que el consumo de bebidas alcohólicas o SPA disminuye las capacidades físicas y mentales para conducir cualquier tipo de vehículo, de ahí la importancia de la detección y penalización de su consumo. En este sentido se reglamentan en leyes de transporte terrestre y seguridad vial, como los ejemplos de Ecuador, Argentina y Colombia, en casos de siniestro o si las autoridades de tránsito lo solicitan ${ }^{52-54}$.

Considerando que el uso de SPA es un problema a nivel mundial, un estudio realizado en Emiratos Árabes Unidos debido al incremento de accidentes de tránsito que involucraba conductores bajo los efectos de las drogas, constató aumentó del uso de dispositivos móviles en las carreteras. Los test en el terreno suelen utilizar fluidos orales, seguido de análisis posteriores de laboratorio para confirmar mediante otra prueba diferente los casos positivos de drogas ilícitas. Es importante para ello cumplir el protocolo de las técnicas de recolección y almacenamiento de las muestras biológicas ${ }^{55}$.

Los dispositivos automatizados para la identificación simultánea de más de una droga como COC, AMP y THC serían de elección ${ }^{56}$, ya que por su alta sensibilidad y especificidad las detecten de manera eficiente en concentraciones bajas de nanogramos por mililitro ${ }^{57}$.

\section{Conclusiones}

La saliva es una matriz usada para la detección de drogas, con ventajas y desventajas respecto a otros fluidos biológicos. Los test de drogas en saliva se basan en diferentes principios físico-químicos, destacando que deben cumplir criterios de sensibilidad, especificidad y umbral de detección adecuados a la identificación de sustancias psicoactivas. Como el consumo implica riesgos personales o contra terceros en el ámbito laboral o en el transporte vehicular por ejemplo, existen regulaciones para el control y la fiscalización de su uso, ante la sospecha de consumo o la vigilancia en centros de tratamiento por dependencia. Los resultados positivos obtenidos mediante test preliminares deben ser confirmados por otra técnica analítica, especialmente si existen consecuencias sanitarias o legales. Si bien los países presentan un marco regulatorio a ese respecto, el análisis de drogas en saliva aún no es de primera elección, dado que se ve dificultado por la falta de estandarización en la obtención y almacenamiento de la muestra y la breve permanencia de las drogas en la saliva.

\section{Referencias bibliográficas}

1. Cadwallader AB, Murray B. Performance-Enhancing Drugs I: Understanding the Basics of Testing for Banned Substances. Int J Sport Nutr Exerc Metab. 2015;25(4):396-404. DOI: 10.1123/ijsnem.2014-0185.

2. Gjerde H, Langel K, Favretto D, Verstraete AG. Detection of 4 benzodiazepines in oral fluid as biomarker for presence in blood. Ther Drug Monit. 2014;36(2):252-6. DOI: 10.1097/FTD.0b013e3182a3ab42.

3. Martini MBA, Batista TBD, Henn IW, Souza PTDR, Vieira AR, Azevedo-Alanis LR. Whether drug detection in urine and oral fluid is similar? A systematic review. Crit Rev Toxicol. 2020;50(4):348-358. DOI: 10.1080/10408444.2020.1751062.

4. Kaczor-Urbanowicz KE, Martin Carreras-Presas C, Aro K, Tu M, Garcia-Godoy F, Wong DT. Saliva diagnostics - Current views and directions. Exp Biol Med (Maywood). 2017;242(5):459-472. DOI: $10.1177 / 1535370216681550$.

5. U.S. National Library of Medicine. Pub Med [İnternet] Drug Testing. [citado 25 de febrero de 2020]. Disponible en: https://medlineplus.gov/lab-tests/drug-testing

6. Truver MT, Palmquist KB, Swortwood MJ. Oral Fluid and Drug Impairment: Pairing Toxicology with Drug Recognition Expert Observations. J Anal Toxicol. 2019;43(8):637-643. DOI: 10.1093/jat/bkz075.

7. Soares Nunes LA, Mussavira S, Sukumaran Bindhu O. Clinical and diagnostic utility of saliva as a non-invasive diagnostic fluid: a systematic review. Biochemia Médica 2015;25(2):177-92.

8. Cone EJ, Huestis MA. Interpretation of oral fluid tests for drugs of abuse. Ann N Y Acad Sci. 2007;1098:51103. DOI: 10.1196/annals.1384.037.

9. Desrosiers NA, Huestis MA. Oral Fluid Drug Testing: Analytical Approaches, Issues and Interpretation of Results. J Anal Toxicol. 2019;43(6):415-443. DOI: 10.1093/jat/bkz048.

10. Lillsunde P. Analytical techniques for drug detection in oral fluid. Ther Drug Monit. 2008;30(2):181-7. DOI: 10.1097/FTD.0b013e3181685088.

11. Cho CM, Hirsch R, Johnstone S. General and oral health implications of cannabis use. Aust Dent J. 
2005;50(2):70-4. DOI: $10.1111 /$ j.1834-7819.2005. tb00343.x.

12. Kintz P. Cannabis et cannabinoïdes de synthèse. À propos de leur détection biologique [Testing for cannabis and synthetic cannabinoids in human specimens]. Bull Acad Natl Med. 2020;204(6):577-582. French. DOI: 10.1016/j.banm.2020.04.003.

13. Zayas Mujica R, Cabrera Cárdenas U, Simón Cayón D. Drogas de abuso: De la embriogénesis a la adolescencia. Rev Cubana Farm [Internet]. 2006 Ago [citado 2020 Mayo 06]; 40(2). Disponible en: http://scielo.sld.cu/scielo.php?script=sci_arttext\&pi$\mathrm{d}=$ S0034-75152006000200011\&lng=es.

14. Pascual Simón JR, Fernández Rodríguez BL. Breve reseña sobre la farmacología de los cannabinoides. MEDISAN [Internet]. 2017 Mar [citado 2021 Jul 11]; 21(3):334345. Disponible en: http://scielo.sld.cu/scielo.php?scrip$\mathrm{t}=$ sci_arttext\&pid=S1029-30192017000300014\&ln$\mathrm{g}=\mathrm{es}$.

15. Mondino A, Sosa S, Zeinsteger P, García y Santos C. Intoxicación por Cannabis en Pequeños Animales. Revisión. Veterinaria (Montev) [Internet]. 2019 Dic [citado 2021 Ene 31]; 55(212): 86-95. Disponible en: http:// www.scielo.edu.uy/scielo.php?script $=$ sci_arttext\&pi$\mathrm{d}=$ S1688-48092019000200086\&lng=es. Epub 01-Dic2019.

16. Gainza I, Nogué S, Martínez Velasco C, Hoffman R. $S$, Burillo-Putze G, Dueñas A et al. Intoxicación por drogas. Anales Sis San Navarra [Internet]. 2003 [citado 2021 Ene 31]; 26(Suppl 1):99-128. Disponible en: http://scielo.isciii.es/scielo.php?script=sci_arttext\&pi$\mathrm{d}=$ S1137-66272003000200006\&lng=es.

17. Denis-Rodríguez E, Hermida-Moreno A. Detección de 3,4- metilenodioximetanfetamina (MDMA) en saliva. Rev Med Mex Forense, 2016;1(1):53-59.

18. NIDA. National Institute on Drug Abuse. [Citado 2019, junio 3]. Abuso y adicción a la metanfetamina. Disponible en: https://www.drugabuse.gov/es/publicaciones/ abuso-y-adiccion-la-metanfetamina en 2020, May 31

19. Götrick B, Giglio D, Tobin G. Effects of amphetamine on salivary secretion. Eur J Oral Sci. 2009;117(3):21823. DOI: $10.1111 /$ j.1600-0722.2009.00629.x.

20. NIDA National Institute on Drug Abuse. [Citado 2017, julio 21]. Los opioides. Disponible en: https://www.drugabuse.gov/es/informacion-sobre-drogas/los-opioides en 2020, May.

21. Woyceichoski IE, Costa $\mathrm{CH}$, de Araújo $\mathrm{CM}$, Brancher JA, Resende LG, Vieira I, de Lima AA. Salivary buffer capacity, $\mathrm{pH}$, and stimulated flow rate of crack cocaine users. J Investig Clin Dent. 2013;4(3):160-3. DOI: 10.1111/j.2041-1626.2012.00126.x.

22. Villanueva J. Matamoros Zelaya M. Ciencias Forenses y Pruebas Presuntivas. Rev. cienc. forenses Honduras. 2016;2(2):45-54.

23. NIH. National Institute of Health [Citado 2020, febrero 25] Pruebas de drogas. Disponible en: https:// medlineplus.gov/spanish/pruebas-de-laboratorio/pruebas-de-drogas/
24. Wang J, Yao W, Meng F, Wang P, Wu Y, Wang B. A surface plasmon resonance immunoassay for the rapid analysis of methamphetamine in forensic oral fluid. J Clin Lab Anal. 2019; 33(9):e22993. DOI:10.1002/jcla.

25. Braithwaite RA, Jarvie DR, Minty PSB, Simpson D, Widdop B. Screening for Drugs of Abuse. I: Opiates, Amphetamines and Cocaine. Ann Clin Biochem. 1995; 32(2):123-153. DOI:10.1177/000456329503200203

26. Naciones Unidas Nueva York. Programa de las Naciones Unidas para la fiscalización internacional de drogas, 1995. Métodos recomendados para la detección y el análisis de heroína, cannabinoides, cocaína, anfetamina, metanfetamina, y derivados anfetamínicos con anillo sustituido en especímenes biológicos. Manual anual para uso de laboratorios nacionales. Disponible en: https://www. unodc.org/documents/scientific/ST-NAR-27-S.pdf

27. Schramm W, Smith RH, Craig PA, Kidwell DA. Drugs of abuse in saliva: a review. J Anal Toxicol. 1992;16(1):19. DOI: $10.1093 /$ jat/16.1.1.

28. Cox KL, Devanarayan V, Kriauciunas A, et al. Immunoassay Methods. 2012 May 1 [Citado 2019 Jul 8]. In: Markossian S, Grossman A, Brimacombe K, et al, editors. Assay Guidance Manual [Internet]. Bethesda (MD): Eli Lilly \& Company and the National Center for Advancing Translational Sciences; 2004. Disponible en: https:/www.ncbi.nlm.nih.gov/books/NBK92434/

29. Naciones Unidas Nueva York, 2012. Métodos recomendados para la identificación y el análisis de cocaína en materiales incautados. Manual para el uso de los laboratorios Nacionales de análisis de estupefacientes.

30. Ochoa Azze RF. Técnicas inmunoenzimáticas para ensayos clínicos de vacunas y estudios inmuno-epidemiológicos. Finlay ediciones. La Habana, 2012.

31. Haldeman Englert C, Walton-Ziegler O. UC San Diego Health. Prueba de detección de cocaína [citado en 9 de enero 2017]. Disponible en: https://myhealth.ucsd.edu/ Spanish/RelatedItems/167,cocaine_screen_ES

32. Verstraete AG. Detection times of drugs of abuse in blood, urine, and oral fluid. Ther Drug Monit. 2004;26(2):2005. DOI: 10.1097/00007691-200404000-00020.

33. Casadiego-Mesa AF, Lastra-Bello SM. Cannabis sintético: aspectos toxicológicos, usos clínicos y droga de diseńo. Rev. Fac. Med. (2015)63(3):501-10. DOI: http:// dx.doi.org/10.15446/revfacmed.v63n3.47460

34. Uruguay. IMPO. Ley de Tránsito y Seguridad Vial. Instituto Nacional de Impresiones y Publicaciones Oficiales IMPO. Ley No18191. Disponible en https://www.impo. com.uy > bases , leyes/18191-2007 (14/11/2007).

35. Uruguay. IMPO. Modificación de la Ley 18.191(Ley de Tránsito y Seguridad Vial). Tolerancia cero de alcohol en sangre. Instituto Nacional de Impresiones y $\mathrm{Pu}-$ blicaciones Oficiales IMPO. Ley No 19360. Disponible en https://www.impo.com.uy/bases/leyes/19360-2015 $(18 / 09 / 2015)$.

36. Uruguay. IMPO. Procedimiento de actuación en materia de consumo de alcohol, cannabis y otras drogas en lugares y ocasión del trabajo. Instituto Nacional de Impresiones y Publicaciones Oficiales IMPO. Decreto No 128/016. Disponible en https://www.impo.com.uy/bases/decretos/128-2016 (02/05/2016). 
37. Pytko-Polonczyk JJ, Jakubik A, Przeklasa-Bierowiec A, Muszynska B. Artificial saliva and its use in biological experiments. J Physiol Pharmacol. 2017; 68(6):807-813.

38. Barriga Angulo G, Hernández Sánchez EA. Utilidad de las muestras de saliva en el diagnóstico por el laboratorio. Rev Latinoam Patol Clin Med Lab. 2016;63(1):13-18.

39. Stein J, Geraedts M. Beigebrauchdetektion in der Substitutionstherapie: Speichelproben versus Urinscreening [Substance abuse detection in substitution therapy: Oral fluid versus urine screening]. Bundesgesundheitsblatt Gesundheitsforschung Gesundheitsschutz. 2017;60(11):1261-1268. German. DOI: 10.1007/ s00103-017-2630-x.

40. Allen KR. Screening for drugs of abuse: which matrix, oral fluid or urine? Ann Clin Biochem 2011;48:531541. DOI: 10.1258/acb.2011.011116

41. Dolan K, Rouen D, Kimber J. An overview of the use of urine, hair, sweat and saliva to detect drug use. Drug Alcohol Rev. 2004;23(2):213-7. DOI: $10.1080 / 09595230410001704208$.

42. Bosker WM, Huestis MA. Oral fluid testing for drugs of abuse. Clin Chem. 2009;55(11):1910-31. DOI: 10.1373/clinchem.2008.108670.

43. Spindle TR, Cone EJ, Herrmann ES, Mitchell JM, Flegel R, LoDico CH, Bigelow GE, Vandrey R. Pharmacokinetics of Cannabis Brownies: A Controlled Examination of $\Delta^{9}$-Tetrahydrocannabinol and Metabolites in Blood and Oral Fluid of Healthy Adult Males and Females. J Anal Toxicol 2020;44(7):661-671. DOI: 10.1093/ jat/bkaa067

44. Weinmann W, Vogt S, Goerke R, Müller C, Bromberger A. Simultaneous determination of THC-COOH and THC-COOH-glucuronide in urine samples by LC/ MS/MS. Forensic Sci Int. 2000;113(1-3):381-7. DOI: 10.1016/s0379-0738(00)00210-3.

45. Casolin A. Comparison of Urine and Oral Fluid for Workplace Drug Testing. J Anal Toxicol. 2016;40(7):479-85. DOI: $10.1093 /$ jat/bkw055.

46. Coulter CA, Moore CM. Analysis of Drugs in Oral Fluid Using LC-MS/MS. Methods Mol Biol. 2019;1872:237259. DOI: 10.1007/978-1-4939-8823-5_22.

47. Rojas SA, Duffau BE, Escobar M. Analysis of Clonazepam in Oral Fluid by SPE-C18 and HPLC-UV-DAD. J Anal Pharm Res. 2017;5(5):00152. DOI: 10.15406/ japlr.2017.05.00152

48. González-Luque JC, Quintela-Jorge O. La determinación de drogas en fluido oral en conductores de vehículos: ¿se abre el camino a la intervención preventiva? Rev Esp Drogodepend. 2011;36(3):341-350.
49. Arroyo Fernández A, Mora Font A, Sánchez Fité M, Barbal Pagés M, Palahí Adroher M. Drogas de abuso en saliva de conductores: aspectos médico-legales. Instituto de Medicina Legal de Cataluña. Barcelona. España. Rev esp med legal. 2008;34(1):3-10.

50. Lyra L, Bellani W, Mazur CE, Brancher JA, Adilson A, Amenábar JM. Changes in salivary composition of chemically dependent subjects. Odontología Vital. 2020;32:63-70.

51. Ochoa Mangado E, Madoz Gúrpide A. Consumo de alcohol y otras drogas en el medio laboral. Med segur trab [Internet]. 2008 [citado 2021 Sep 19]; 54(213):25-32. Disponible en: http://scielo.isciii.es/scielo.php?script=sci_arttext\&pid=S0465-546X2008000400003\&lng=es.

52. Ecuador. Reglamento a ley de transporte terrestre, tránsito y seguridad vial. Decreto Ejecutivo 1196 Registro Oficial Suplemento 731 de 25-jun-2012. Disponible en: https://www.obraspublicas.gob.ec/wp-content/uploads/ downloads/2015/03/Decreto-Ejecutivo-No.-1196-de11-06-2012-reglamento-a-la-ley-de-transporte-terrestretransito-y-seguridad-via.pdf

53. Argentina. Ministerio de Justicia y Derechos $\mathrm{Hu}-$ manos. Presidencia de la Nación. Tránsito y Seguridad Vial. Ley 26.363, abril de 2008. Disponible en: http://servicios.infoleg.gob.ar/infolegInternet/anexos/140000-144999/140098/texact.htm

54. Colombia. Poder Público-Rama Legislativa. Ley 769 de 2002 "Por la cual se expide el Código Nacional de Tránsito Terrestre y se dictan otras disposiciones". Disponible en: https://www.movilidadbogota.gov.co/web/ sites/default/files/ley-769-de-2002-codigo-nacional-detransito_3704_0.pdf

55. Alhefeiti MA, Barker J, Shah I. Roadside Drug Testing Approaches. Molecules. 2021;26(11):3291. DOI: $10.3390 /$ molecules 26113291

56. Risoluti R, Gullifa G, Battistini A, Materazi S. Anal. Chem. (2019)91(10):6435-6439. DOI:10.1021/acs. analchem.9b00197

57. Reinstadler V, Lierheimer S, Boettcher $\mathrm{M}$ et al. A validated workflow for drug detection in oral fluid by non-targeted liquid chromatography-tandem mass spectrometry. Anal Bioanal Chem. 2019;411:867-876. DOI: $10.1007 /$ s00216-018-1504-x 